\title{
Atrial Thrombus in a Neonate: A Diagnostic Challenge
}

\author{
Alicia Sheen, MD ${ }^{1}$ Elizabeth R. De Oliveira, $\mathrm{MD}^{2,3}$ \\ Ashwini Lakshmanan, MD, MPH ${ }^{6}$ \\ ${ }^{1}$ Division of General Pediatrics, Children's Hospital Los Angeles, Keck \\ School of Medicine at the University of Southern California, Los \\ Angeles, California \\ 2 Pacific Pediatric Cardiology Medical Group, Inc., Los Angeles, California \\ ${ }^{3}$ Division of Cardiology, Children's Hospital Los Angeles, Keck School of \\ Medicine at the University of Southern California, Los Angeles, California \\ ${ }^{4}$ Division of Cardiothoracic Surgery, Children's Hospital Los Angeles, \\ Keck School of Medicine at the University of Southern California, Los \\ Angeles, California \\ ${ }^{5}$ Division of Pathology and Laboratory Medicine, Children's Hospital \\ Los Angeles, Keck School of Medicine at the University of Southern \\ California, Los Angeles, California \\ ${ }^{6}$ Division of Newborn and Infant Critical Care, Children's Hospital Los \\ Angeles, Keck School of Medicine at the University of Southern \\ California, Los Angeles, California
}

Richard W. Kim, MD, FACS ${ }^{4}$ David Parham, MD ${ }^{5}$

Am J Perinatol Rep 2015;5:e18-e21.

\begin{abstract}
Introduction Left atrial thrombus is a rare finding in a neonate. In the previous literature, atrial thrombi have been associated with catheter placement or congenital heart disease in a preterm infant.

Case We report the case of a full-term neonate with no known risk factors found to have a left atrial thrombus. The neonate was born at 38 weeks' gestation to a 31-year-old female via cesarean section who was sent to the normal nursery. On postnatal day 5 , the infant was noted to have low-to-medium level of oxygen saturations $(\sim 90 \%)$ and was transferred to the neonatal intensive care unit with an echocardiogram completed on postnatal day 6 demonstrating a mobile, pedunculated mass attached to the left atrial septum with an appearance concerning for atrial myxoma. The infant underwent surgical resection on postnatal day 8 and pathology revealed the mass to be a left atrial thrombus.
Keywords
- atrial thrombus
- neonate
- infant
- thrombus resection
- heparin

Discussion The rare finding of an atrial thrombus in a neonate has previously been associated with central venous catheter placement or congenital heart disease. This case is unusual in that the patient had neither condition. Although echocardiogram findings appeared more consistent with atrial myxoma, final pathology revealed a left atrial thrombus. Additionally, hypercoagulability work-up for this neonate was largely negative. This report underscores the importance of identification, search for etiology, and prompt therapy to prevent potential catastrophic outcomes.
\end{abstract}

Address for correspondence Ashwini Lakshmanan, MD, MPH, Division of Newborn and Infant Critical Care, Children's Hospital Los Angeles, Sunset Blvd, MS \#31, Los Angeles, CA 90027 (e-mail: alakshmanan@chla.usc.edu).
Neonatal atrial thrombus has been reported only a handful of times in the literature thus far. The incidence of symptomatic neonatal arterial thromboembolic disease is reported to be approximately 0.25 of 10,000 live births with $90 \%$ of these associated with arterial access devices. Mortality is high, irrespective of treatment and has been as high as 33\% received

July 29, 2014

accepted after revision

October 14, 2014

published online

January 16, 2015
DOI http://dx.doi.org/

10.1055/s-0034-1396567. ISSN 2157-6998.
Copyright $\odot 2015$ by Thieme Medical Publishers, Inc., 333 Seventh Avenue, New York, NY 10001, USA. Tel: +1(212) 584-4662.
License terms

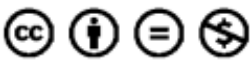


in neonates with aortic thrombosis. ${ }^{1}$ Atrial thrombus is typically associated with either catheter placement, specific structural abnormalities such as congenital heart disease, or hypercoagulability states. Ina et al describes the case of a neonatal atrial thrombus detected secondary to late-onset circulatory dysfunction and reports a total of six reported cases in the literature of atrial thrombi before that case. ${ }^{2-6}$ Atrial thrombi are usually detected by an echocardiogram obtained in infants following a deep insertion of a catheter into the atrium. In other cases, known congenital heart disease, such as mitral valve stenosis, leads to left atrial blood congestion that helps in obtaining an echocardiogram. ${ }^{7}$ In this case report, we describe a case of a full-term neonate with no known risk factors found to have left atrial thrombus.

\section{Case Report}

A female infant was born at 38 weeks' gestation to a 31-year-old G3P2Ab1 female who had prenatal care. The neonate was born via cesarean section secondary to fibroid uterus, with an APGAR score of 9 at 1 and 5 minutes, and did not require resuscitation. In the regular nursery, the infant was noted to be jaundiced on postnatal day 1 with a bilirubin of $10.4 \mathrm{mg} / \mathrm{dL}$. She was started on phototherapy for a 4-day course. The infant also received a 5-day course of ampicillin and gentamicin secondary to unknown maternal group B streptococcus status and elevated C-reactive protein of $3.98 \mathrm{mg} / \mathrm{dL}$. Blood and urine cultures obtained before initiating antibiotics were negative.

On postnatal day 5 , the infant was noted to have low-tomedium level of oxygen saturations $(\sim 90 \%)$ and was transferred to the neonatal intensive care unit and placed on $0.5 \mathrm{~L}$ per minute of oxygen via nasal cannula to maintain saturations $>95 \%$. A chest radiograph demonstrated bilateral haziness at the bases, concerning for possible pulmonary edema. An echocardiogram was obtained on postnatal day 6 that demonstrated a structurally normal heart with a mobile, pedunculated mass with a narrow stalk attached to the left atrial septum. The infant was then transferred to a tertiary center for surgical intervention. She was started on a heparin drip at 28 units $/ \mathrm{kg} / \mathrm{h}$ on postnatal day 7 and continued for 15 hours. A repeat echocardiogram on postnatal day 7 confirmed a large, pedunculated, well defined, mobile mass measuring approximately $7 \times 12 \mathrm{~mm}$ with a narrow stalk attached to the left atrial septum at the level of the fossa ovalis, concerning for atrial myxoma versus thrombus (-Fig. 1A, B).

Preoperative transesophageal echo performed on postnatal day 8 demonstrated a $6 \times 6 \times 10 \mathrm{~mm}$ echo bright mass tethered to redundant septum primum, more consistent in appearance and location to an atrial myxoma than thrombus. The mass did not appear to interfere with mitral function or inflow. The neonate underwent resection of the left atrial mass with no intraoperative complications. Postoperative transesophageal echocardiogram showed that the mass had been successfully resected and that the left atrial appendage was completely clear. Subsequent pathology analysis revealed the mass to be a thrombus ( - Fig. 2). The postoperative course was uncomplicated, and the neonate was extubated and a

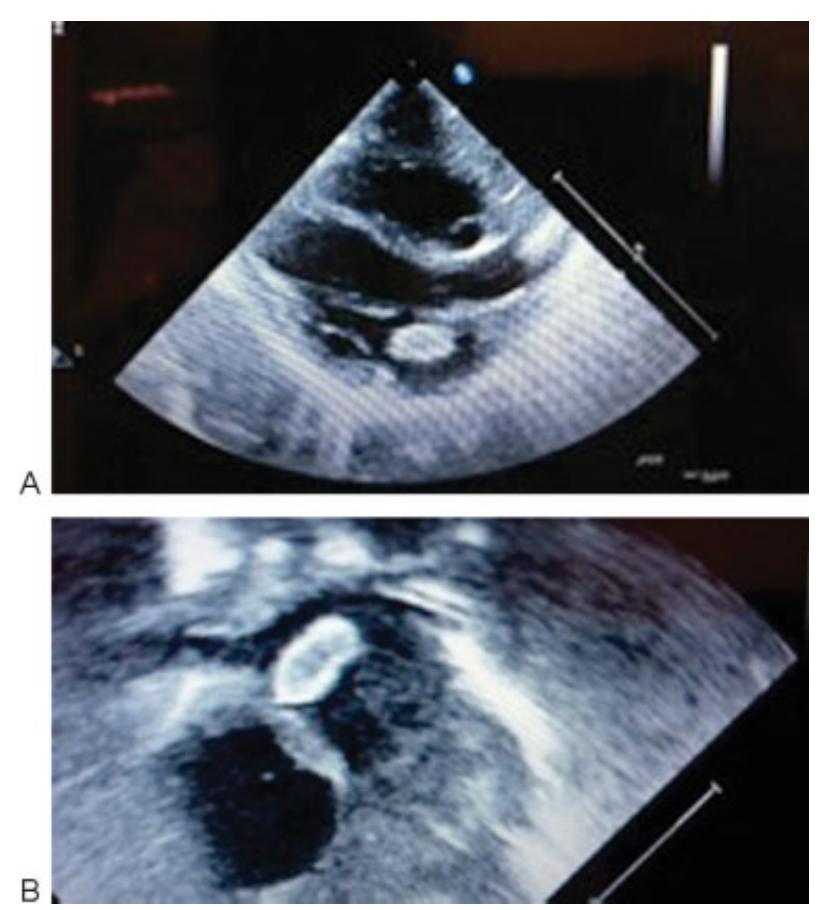

Fig. 1 ( $A$ and $B$ ) Parasternal long-axis view of transthoracic echocardiogram and apical four-chamber view of transthoracic echocardiogram of atrial thrombus. Large, pedunculated, well-defined, mobile mass measuring approximately $7 \times 12 \mathrm{~mm}$ with a narrow stalk attached to the left atrial septum at the level of the fossa ovalis.

mediastinal chest tube was removed on postoperative day 1 (-Figs. 3 and 4 ).

Hematological workup included protein C level, protein $\mathrm{S}$ level, antithrombin III level, prothrombin gene mutation, homocysteine level, and antiphospholipid antibody panel. The initial platelet count was $287 \mathrm{~K} / \mathrm{L}^{\mathrm{L}}$, and there were no signs of consumptive coagulopathy. All laboratories were within normal range except an equivocal homocysteine level of $18 \mu \mathrm{mol} / \mathrm{L}$ (range, $2.5-12.5 \mu \mathrm{mol} / \mathrm{L}$ ). Aspirin was started at a daily dose of $40.5 \mathrm{mg}$ by mouth on postnatal day 11 . At a follow-up hematology, outpatient visit on postnatal day 31, the neonate remained on aspirin and appeared healthy and

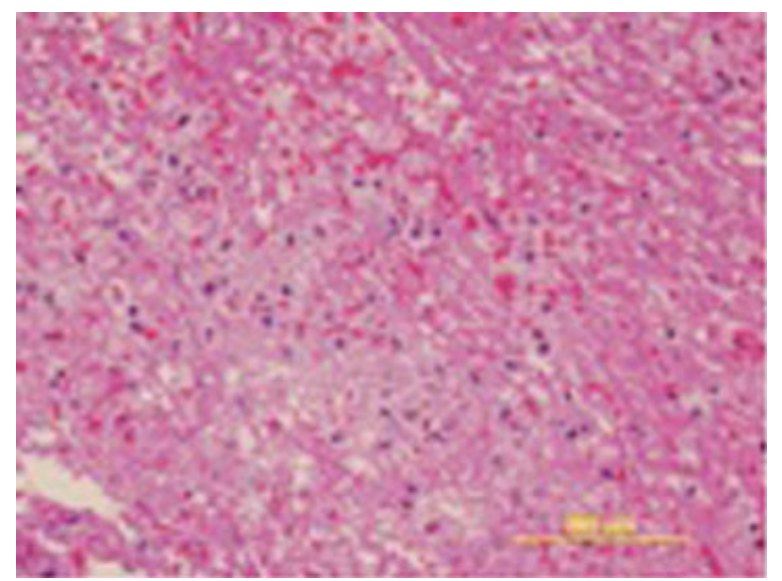

Fig. 2 Hematoxylin and eosin stain of soft tissue mass excision from left atrium, consistent with fibrin and red blood cell mural thrombus. 


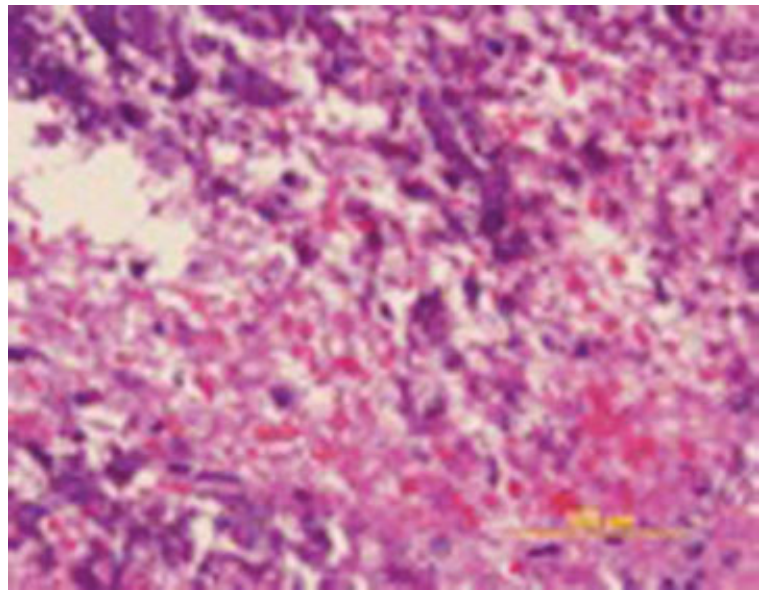

Fig. 3 Hematoxylin and eosin stain of a soft tissue mass excision from the left atrium that now demonstrates calcification.

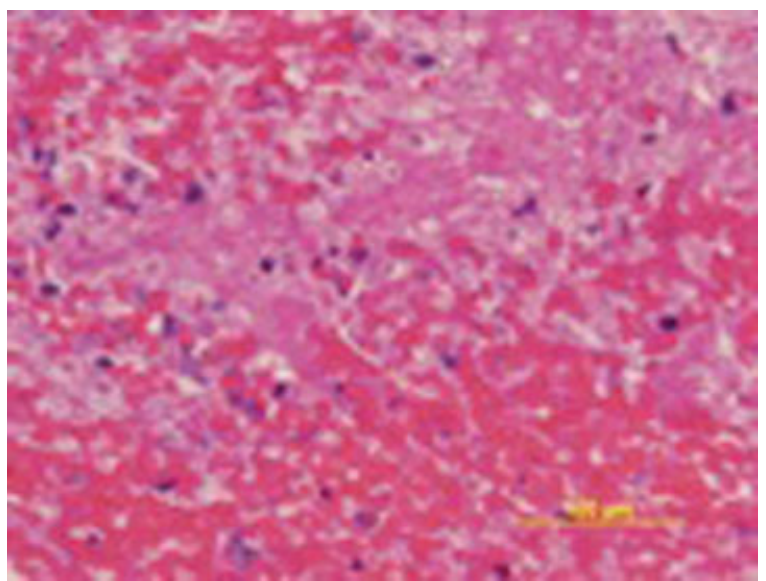

Fig. 4 Hematoxylin and eosin stain of a soft tissue mass excision from the left atrium that demonstrates early organization indicating chronicity and suggests a possible relation to foramen ovale turbulence.

asymptomatic. Follow-up transthoracic echocardiograms performed 1 and 6 months postoperative revealed no recurrence of thrombus formation. Aspirin was then stopped.

\section{Discussion}

Atrial thrombus is exceedingly rare in the neonate. On review of the available literature, most atrial thrombi are related to placement of central venous catheters or congenital heart disease. ${ }^{2}$ This case is unusual in that the patient did not have a central catheter placed nor evidence of congenital heart disease. In this case, atrial thrombus was detected unexpectedly by echocardiogram that was ordered secondary to mild respiratory distress.

Another interesting observation in this case is that the initial echocardiogram findings were more consistent in location and appearance with a myxoma rather than a thrombus. The infant's echocardiogram demonstrated a large, pedunculated mobile mass with a narrow stalk attached to the left atrial septum at the level of the fossa ovalis, as is consistent with the typical appearance of myxoma tumors on echocardiogram. ${ }^{8}$ Because of the location of the tumor and concerns for embolization, the decision was made to have it resected. On resection, cardiac pathology revealed a final diagnosis of a thrombus rather than atrial myxoma. Histopathology did not demonstrate the typical appearance of myxoma cells arranged singly, in cords, or in vasoformative ring structures with elongated, fusiform, or stellate cells. ${ }^{8}$ Instead, histopathology revealed fibrin and red blood cells consistent with a left atrial mural thrombus. Scheffel et al performed a study that evaluated 23 adult patients with atrial myxomas and thrombi to compare distinguishing features of atrial myxomas versus thrombi using computed tomography and found size, shape, origin, mobility, and prolapse to be helpful features in distinguishing

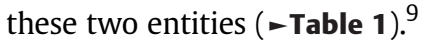

Also notable in this case was that work-up for hypercoagulability as a possible underlying etiology for neonatal atrial thrombus was largely negative. However, this particular neonate did have a mildly elevated homocysteine value of $18 \mu \mathrm{mol} / \mathrm{L}$. Normal neonatal laboratory range for homocysteine is approximated from 2.5 to $12.5 \mu \mathrm{mol} / \mathrm{L}$. The clinical significance of this mild elevation is unclear at this time; hematology deemed this value "equivocal" and recommended a follow-up homocysteine level. Prior study performed by Kenet et al used a value of homocysteine $>15$ $\mu \mathrm{mol} / \mathrm{L}$ in their investigation of thrombophilia and neonatal complications, but they did not find any association between values deemed as "thrombophilic" and preterm infant complications. $^{10}$

This report underscores the importance of identification, search for etiology, and prompt therapy to prevent potential catastrophic outcomes. In this case, heparin drip, cardiothoracic resection of the atrial mass followed by long-term aspirin therapy was effective and provided good outcome

Table 1 Comparison of imaging features on CT of atrial myxomas and thrombi compiled by Scheffel et al $^{9}$

\begin{tabular}{|l|l|l|}
\hline Characteristic & Myxoma & Thrombi \\
\hline Mean size, $\mathrm{mm}$ & $33.0 \pm 16.4$ & $21.2 \pm 7.3$ \\
\hline Shape & Some polypoid, some villous & Polypoid, no villous \\
\hline Origin & Fossa ovalis, interatrial septum, posterior or lateral wall & Left atrial appendage, posterior or lateral wall \\
\hline Mobility & More mobile & Less mobile \\
\hline Prolapse & Prolapse & No prolapse \\
\hline
\end{tabular}


for this patient. Similar treatment in the hands of an experienced team may be of use for future patients who present in a similar manner.

\section{Contributions}

Dr. Sheen made substantial contributions to designing the case report and wrote the first draft of the article. Drs. Kim and Parham revised the article. Drs. Lakshmanan and De Oliveira made substantial contributions in designing the case report and critically revising the article. All the authors reviewed and approved the final version of the article.

Funding

No external funding was received for this article.

Financial Disclosure

None.

\section{References}

1 Kenny D, Tsai-Goodman B. Neonatal arterial thrombus mimicking congenital heart disease. Arch Dis Child Fetal Neonatal Ed 2007; 92(1):F59-F61
2 Ina $\mathrm{S}$, Futatani $\mathrm{T}$, Higashiyama $\mathrm{H}$, et al. Left atrium thrombus in an extremely low-birthweight infant with late-onset circulatory dysfunction. Pediatr Int 2012;54(4):555-558

3 Ferrari F, Vagnarelli F, Gargano G, et al. Early intracardiac thrombosis in preterm infants and thrombolysis with recombinant tissue type plasminogen activator. Arch Dis Child Fetal Neonatal Ed 2001;85(1):F66-F69

4 Aspesberro F, Beghetti M, Oberhänsli I, Ozsahin H, Humbert J, Rimensberger PC. Local low-dose urokinase treatment of acquired intracardiac thrombi in preterm infants. Eur J Pediatr 1999;158(9): 698-701

5 Al Dhahri KN, Sandor GG, Duncan WJ. Intra-atrial thrombus in a neonate with coarctation of the aorta. Cardiol Young 2006;16(4): 392-394

6 Romano A, Weinberg PM, Woolf PK, Vetter VL. Pulmonary venous obstruction from left atrial thrombus in hypoplastic left heart syndrome. Pediatr Cardiol 1989;10(2):105-107

7 Beppu S, Nimura Y, Sakakibara H, Nagata S, Park YD, Izumi S. Smoke-like echo in the left atrial cavity in mitral valve disease: its features and significance. J Am Coll Cardiol 1985;6(4):744-749

8 World Health Organization Classification of Tumours. Pathology and genetics of tumours of the lung, pleura, thymus and heart. In: William D. Travis, Elizabeth Brambilla, H. Konrad, Muller-Hermelink, and Curtis C. Harris eds. WHO Publications Center. Lyon: IARC Press; 2004:260-263

9 Scheffel H, Baumueller S, Stolzmann P, et al. Atrial myxomas and thrombi: comparison of imaging features on CT. AJR Am J Roentgenol 2009;192(3):639-645

10 Kenet G, Maayan-Metzger A, Rosenberg N, et al. Thrombophilia does not increase risk for neonatal complications in preterm infants. Thromb Haemost 2003;90(5):823-828 